Fukushima J. Med. Sci.,

Vol. 64, No. 1, 2018

\title{
[Original Article]
}

\section{Comparison of surgical outcomes after pneumonectomy and pulmonary function-preserving surgery for non-small cell lung cancer}

\author{
Mitsunori Higuchi ${ }^{1)}$, Hironori Takagi ${ }^{2)}$, Yuki Ozaki ${ }^{2)}$, Takuya Inoue ${ }^{2)}$, Yuzuru Watanabe ${ }^{2)}$, \\ Takumi Yamaura ${ }^{2)}$, Mitsuro Fukuhara ${ }^{2)}$, Satoshi Muto ${ }^{2)}$, Naoyuki Okabe ${ }^{2)}$, Yuki Matsumura ${ }^{2)}$, \\ Takeo Hasegawa ${ }^{2)}$, Jun Osugi ${ }^{2)}$, Mika Hoshino ${ }^{2)}$, Yutaka Shio ${ }^{2)}$ and Hiroyuki Suzuki ${ }^{2)}$ \\ ${ }^{1)}$ Department of Thoracic Surgery, Aizu Medical Center, Fukushima Medical University, Aizuwakamat- \\ su, Japan, ${ }^{2)}$ Department of Chest Surgery, Fukushima Medical University School of Medicine, Fukushi- \\ ma, Japan
}

(Received May 8, 2017, accepted January 24, 2018)

\begin{abstract}
Background : According to previous reports, lobectomy with bronchoplasty or angioplasty is a more feasible surgery than pneumonectomy for central-type non-small cell lung cancer. However, few studies have compared both the short- and long-term outcomes between pneumonectomy and pulmonary function-preserving surgery.

Methods : From January 2004 to December 2015, 18 patients underwent pneumonectomy (Group $\mathrm{PN}$ ) and 12 patients underwent pulmonary function-preserving surgery (group PS) at Fukushima Medical University Hospital. Clinicopathological factors were statistically compared between the two groups.

Results : The operation times in Group PN and Group PS were 285.9 \pm 27.9 and $271.3 \pm 99.2$ min, respectively $(p=0.613)$, while the amounts of intraoperative bleeding were $324.8 \pm 248.9$ and $164.5 \pm 116.6$ g, respectively $(p=0.020)$. The duration of chest drainage and hospitalization after surgery in both groups were not significantly different but there was a tendency toward shorter periods of these durations in Group PS. The 5-year disease-free survival (DFS) rate in Group PN and PS was $51.4 \%$ and $74.1 \%$, respectively, without a significant difference $(p=0.298)$. The 5 -year overall survival (OS) rate in Group PN and PS was 52.5\% and 56.6\%, respectively, also without a significant difference $(p=0.748)$. The 5 -year OS rate was inferior to the 5 -year DFS rate in Group PS, and the 5-year OS rate was not better than the 5-year DFS rate in Group PN.

Conclusions : The short-term results were better in Group PS than PN. However, the long-term results in both groups were similar. Other causes of death influenced OS in both groups ; this result might have been affected by the surgical procedures.
\end{abstract}

Key words : Pneumonectomy, Bronchoplasty, Angioplasty, Surgical outcomes, Non-small cell lung cancer (NSCLC)

\section{Introduction}

Central-type lung cancer sometimes invades bronchial openings and/or the pulmonary artery $(\mathrm{PA})$. For these patients, lobectomy/segmentectomy with bronchoplasty or PA angioplasty is often preferred. This surgery sometimes requires simul- taneous reconstruction of the airways and/or blood vessels. On the other hand, pneumonectomy for lung cancer is reportedly associated with significant morbidity and mortality ${ }^{1-3)}$, including postpneumonectomy lung edema, adult respiratory distress syndrome, bronchopleural fistula, and postpneumonectomy syndrome ${ }^{3)}$. We recently began to employ

Corresponding author : Mitsunori Higuchi, M.D., Ph.D. E-mail : higuchi@fmu.ac.jp

https://www.jstage.jst.go.jp/browse/fms http://www.fmu.ac.jp/home/lib/F-igaku/ 
pulmonary function-preserving surgery to avoid morbidity and mortality whenever technically possible. However, pneumonectomy is inevitable when complete resection cannot otherwise be achieved. Previous reports have already shown that lobectomy with bronchoplasty or angioplasty is a more feasible surgery than pneumonectomy for central-type nonsmall cell lung cancer (NSCLC). However, these previous reports independently compared the outcomes between pneumonectomy and lobectomy with bronchoplasty or between pneumonectomy and lobectomy with angioplasty. Few reports have compared the short- and long-term outcomes between pneumonectomy and pulmonary functionpreserving surgery including both lobectomy with bronchoplasty and with angioplasty together when performed to avoid pneumonectomy. In this study, we evaluated these clinicopathological results to reveal the utility of pulmonary function-preserving surgery.

\section{Materials and Methods}

\section{Patient characteristics}

From January 2004 to December 2015, pulmonary resection was performed in 1150 consecutive patients with primary NSCLC at Fukushima Medical University Hospital in Japan. Of these patients, 18 (1.6\%) underwent pneumonectomy (Group PN) and $12(1.0 \%)$ underwent pulmonary function-preserving surgery (lobectomy with bronchoplasty, $n=5$; lobectomy with angioplasty, $n=5$; and lobectomy with both bronchoplasty and angioplasty, $n=2$; Group PS). The patients' clinical records were retrospectively analyzed, and further data were collected from patient surveys. Variables were shown as mean \pm standard deviation (SD). The protocol was conducted in accordance with the Declaration of Helsinki and Good Clinical Practice guidelines.

\section{Preoperative staging}

The indications for surgery, clinical staging work-up, preoperative cardiopulmonary function tests, possible induction therapy, and postoperative course were evaluated. The preoperative work-up included a clinical history, physical examination, and chest computed tomography (CT) scan with intravenous contrast no more than 1 month before surgery as well as a complete blood count, biochemical blood analysis, and blood gas analysis. Cardiovascular and respiratory function was assessed by electrocardiography and pulmonary function tests. When a patient was suspected to have N2 disease based on CT or fluorodeoxyglucose-positron emission tomography findings, transbronchial needle aspiration biopsy was performed. Platinum-based induction chemotherapy was administered in patients with $\mathrm{N} 2$ disease.

\section{Surgical procedures}

The procedures were performed under general anesthesia with double-lumen tube placement through a standard posterolateral thoracotomy. The indications for the vascular reconstructive procedure and bronchoplastic procedure were direct PA infiltration by the tumor or metastatic lymph nodes and bronchial infiltration by the tumor or metastatic lymph nodes, respectively. The indication for pneumonectomy was direct PA or bronchial infiltration and pulmonary invasions to the ipsilateral lobes. If the main PA was macroscopically free from cancer involvement and sufficiently distant from the tumor, both proximal and peripheral control of the main PA was achieved using a Satinsky clamp. Once the lobe had been removed, a fine measurement of the vascular defect was conducted. If the defect comprised $>25 \%$ of the anterior wall, we used the pericardium to achieve satisfactory functional anatomy of the vessel. The bronchial margins were confirmed to be tumor-free by frozen histologic evaluation. End-to-end bronchial anastomosis was performed with interrupted absorbable 3-0 or 4-0 sutures (PDS II ; Ethicon, Inc., Somerville, NJ, USA). All patients underwent curative oncologic resection associated with complete mediastinal lymphadenectomy. After thoracotomy closure, pain was controlled by means of continuous epidural ropivacaine hydrochloride hydrate or intravenous fentanyl.

Surgical pathologic examination was conducted using morphologic and immunohistochemical techniques and evaluated according to the standard of Union for International Cancer Control $7^{\text {th }}$ edition, 2009.

\section{Treatment and follow-up}

Patients who underwent lobectomy with angioplasty were followed up postoperatively by contrast CT with multi-planar reconstruction at 1 week postoperatively and again at 3 to 6 months postoperatively. Patients who underwent pneumonectomy and lobectomy with bronchoplasty were followed-up postoperatively by bronchoscopy and CT at 1 to 3 months postoperatively to observe the healing of the bronchial stump and anastomosis. For the next 24 
months, all patients underwent chest X-ray examinations every 1 month and contrast CT examinations every 6 months. CT scans were then scheduled every 12 months if the patients were formerly negative for cancer relapse. Locoregional recurrence was defined as recurrence of disease at the resection margin, bronchial stump, or ipsilateral hemithorax. Distant recurrence included the supraclavicular fossa, contralateral hilum, and all other distant organs.

\section{Statistical analysis}

Clinicopathological factors were compared between the two groups using the two-tailed Pearson's chi-square test. Fisher's exact test was used to analyze categorical variables, and the t-test was used for continuous variables. Survival probabilities were estimated using the Kaplan-Meier method. All tests were two-sided, and a $p$ value of $<0.05$ was considered statistically significant. Statistical analysis was conducted with SPSS version 21.0 (IBM Corp., Armonk, NY, USA).

\section{Results}

\section{Patient characteristics}

Comparison of the patients' clinicopathological characteristics and operative results in Group PN $(n=18)$ and Group PS $(n=12)$ are shown in Table 1. There were no significant differences in age, sex, affected side, or tumor size between the two groups ; however, the histologic type trended toward squamous cell carcinoma in Group PN and non-squamous cell carcinoma in Group PS $(p=0.073)$. The Brinkmann index was higher in Group PS than PN (941.7 \pm 434.3 and $1253 \pm 762.5$, respectively ; $p=$ 0.030 ). The use of induction chemotherapy and adjuvant chemotherapy were not significantly different between the two groups. Each group had similar preoperative pulmonary function. The mean preoperative forced expiratory volume in 1 second for patients who underwent pneumonectomy was $2091 \pm 126.3 \mathrm{ml}$, and that in patients who underwent pulmonary function-preserving surgery was $2316 \pm 125.9 \mathrm{ml}(p=0.914)$. These data show that all patients had tolerability for the surgical treatment. In both groups, the bronchus and/or PA was involved by the primary tumor or metastatic lymph nodes. In Group PN, pulmonary invasion to other lobes was considered the indication for pneumonectomy, whereas pulmonary invasion was not found in Group PS.

\section{Short-term results}

To evaluate the short-term clinical results, the following parameters were compared between the two groups: operation time, intraoperative bleeding, duration of chest drainage, duration of hospitalization, operative mortality, and operative morbidity (Table 2). The operation time in Group PN and PS were $285.9 \pm 27.9$ and $271.3 \pm 99.2 \mathrm{~min}$, respectively $(p=0.613)$, while the amount of intraoperative bleeding in the two groups was $324.8 \pm 248.9$ and $164.5 \pm$ $116.6 \mathrm{~g}$, respectively $(p=0.020)$. The duration of chest drainage and hospitalization after surgery in Group PN were $9.8 \pm 15.5$ and $26.9 \pm 33.9$ days, respectively, and those in Group PS were $3.2 \pm 2.6$ and $10.2 \pm 3.7$ days, respectively, with a tendency toward a shorter period in Group PS ( $p=0.055$ and 0.074 , respectively). Operative mortality was defined as any death within 30 days of surgery or during the same hospital admission. Only one case of operative mortality occurred in this study (Group PN). However, 13 cases of operative morbidity (72.2\%) occurred in Group PN and 4 (33.3\%) occurred in Group PS, with a significant difference between the two groups $(p=0.035)$. Common complications after surgery included early- or late-onset bronchial fistula $(n=3)$, pneumonia $(n=2)$, chylotho$\operatorname{rax}(n=2)$, and pyothorax $(n=2)$ in Group PN and vocal cord palsy $(n=2)$, pneumonia $(n=1)$, prolonged air leakage $(n=1)$, and delirium $(n=1)$ in Group PS.

\section{Long-term results}

The median follow-up time was $42.9 \pm 39.7$ months ( range, 1-127 months). We checked the final assessment of survival in July 31, 2016). During the follow-up period, recurrence occurred in $38.9 \%$ (7 of 18 patients) in Group PN (locoregional recurrence in 1, distant metastasis in 4, and locoregional recurrence + distant metastasis in 2) and $25.0 \%$ (3 of 12 patients) in Group PS (distant metastasis in 2 and locoregional recurrence + distant metastasis in 1 ). There were no significant differences in recurrence between the two groups $(p=0.791)$ (Table 3). The 5-year disease-free survival (DFS) rate was $51.4 \%$ and $74.1 \%$ in Group PN and PS, respectively, without a significant difference $(p=0.298)$ (Figure 1). The 5 -year overall survival (OS) rate was $52.5 \%$ and $56.6 \%$ in Group PN and PS, respectively, also without a significant difference $(p=0.748)$ (Figure 2). Remarkably, the 5-year OS was inferior to the 5-year DFS in Group PS, and the 5-year OS in Group PN was not better than the 5-year DFS in Group PN. The causes of death in Groups PN and 
Table 1. Patient characteristics

\begin{tabular}{|c|c|c|c|}
\hline Characteristics & Group PN ( $n=18)$ & Group PS $(n=12)$ & $p$ value \\
\hline Age (y) & $66.10 \pm 6.13$ & $68.70 \pm 6.02$ & 0.755 \\
\hline Sex & & & 0.232 \\
\hline Male & $16(88.9 \%)$ & $12(100.0 \%)$ & \\
\hline Female & $2(11.1 \%)$ & $0(0.0 \%)$ & \\
\hline Brinkmann index & $941.7 \pm 434.3$ & $1253 \pm 762.5$ & 0.030 \\
\hline Histology & & & 0.073 \\
\hline $\mathrm{Sq}$ & $12(66.7 \%)$ & $4(33.3 \%)$ & \\
\hline Non-Sq & $6(33.3 \%)$ & $8(66.7 \%)$ & \\
\hline Side & & & 0.216 \\
\hline Left & $13(72.2 \%)$ & $6(50.0 \%)$ & \\
\hline Right & $5(27.8 \%)$ & $6(50.0 \%)$ & \\
\hline Site & & & 0.037 \\
\hline Upper lobe & $9(50.0 \%)$ & $11(91.7 \%)$ & \\
\hline Middle lobe & $2(11.1 \%)$ & $1(8.3 \%)$ & \\
\hline Lower lobe & $7(38.9 \%)$ & $0(0.0 \%)$ & \\
\hline Preoperative FEV1 (ml) & $2091 \pm 126.1$ & $2317 \pm 125.9$ & 0.914 \\
\hline Tumor size (mm) & $55.1 \pm 4.54$ & $39.3 \pm 5.24$ & 0.934 \\
\hline Induction therapy & & & 0.511 \\
\hline Chemotherapy & $3(16.7 \%)$ & $1(8.3 \%)$ & \\
\hline Radiotherapy & $0(0.0 \%)$ & $0(0.0 \%)$ & \\
\hline None & $15(83.3 \%)$ & $11(91.7 \%)$ & \\
\hline p-Nodal status & & & 0.667 \\
\hline No & $8(44.4 \%)$ & $5(41.7 \%)$ & \\
\hline N1 & $5(27.8 \%)$ & $5(41.7 \%)$ & \\
\hline $\mathrm{N} 2$ & $5(27.8 \%)$ & $2(16.6 \%)$ & \\
\hline p-Stage & & & 0.095 \\
\hline $\mathrm{IA} / \mathrm{IB}$ & $0 / 3$ & $3 / 0$ & \\
\hline IIA/IIB & $3 / 3$ & $2 / 3$ & \\
\hline IIIA/IIIB & $9 / 0$ & $3 / 1$ & \\
\hline Invasion of tumor or LNs & & & 0.075 \\
\hline PA involvement & $6(33.3 \%)$ & $5(41.7 \%)$ & \\
\hline Br involvement & $6(33.3 \%)$ & $5(41.7 \%)$ & \\
\hline $\mathrm{PA}$ and $\mathrm{Br}$ involvement & $0(0.0 \%)$ & $2(16.6 \%)$ & \\
\hline Other lobes invasion & $6(33.3 \%)$ & $0(0.0 \%)$ & \\
\hline Reconstruction & & & - \\
\hline Bronchoplasty & - & $5(41.7 \%)$ & \\
\hline Angioplasty & - & $5(41.7 \%)$ & \\
\hline Both & - & $2(16.6 \%)$ & \\
\hline Adjuvant therapy & & & 0.255 \\
\hline Chemotherapy & $2(11.1 \%)$ & $5(41.7 \%)$ & \\
\hline Radiotherapy & $2(11.1 \%)$ & $0(0.0 \%)$ & \\
\hline None & $14(77.8 \%)$ & $7(58.3 \%)$ & \\
\hline
\end{tabular}

Sq, squamous cell carcinoma

Non-Sq, non-squamous cell carcinoma

FEV1, forced expiratory volume in 1 second

LNs, lymph nodes

PA. pulmonary artery

$\mathrm{Br}$, bronchus 
Table 2. Postoperative morbidity and mortality

\begin{tabular}{lccc}
\hline & Group PN $(n=18)$ & Group PS $(n=12)$ & $p$ value \\
\hline Operation time (min) & $286 \pm 27.9$ & $271 \pm 99.2$ & 0.613 \\
Bleeding during surgery $(\mathrm{g})$ & $325 \pm 249$ & $165 \pm 117$ & 0.020 \\
Duration of chest drainage (days) & $9.8 \pm 15.5$ & $3.2 \pm 2.6$ & 0.055 \\
Postoperative hospitalization (days) & $26.9 \pm 33.9$ & $10.2 \pm 3.7$ & 0.074 \\
Operative mortality & $1(5.6 \%)$ & $0(0.0 \%)$ & 0.406 \\
Operative morbidity & $13(72.2 \%)$ & $4(33.3 \%)$ & 0.035 \\
Complications (total number) & 14 & 5 & \\
Bronchial fistula & 3 & 0 & \\
Pneumonia & 2 & 2 & \\
Hoarseness & 1 & 0 & \\
Chylothorax & 2 & 0 & \\
Pyothorax & 2 & 0 & \\
SVC thrombosis & 1 & 0 & \\
Pericarditis & 1 & 0 & \\
Anemia & 1 & 0 & \\
Arrhythmia & 1 & 0 & \\
Interstitial pneumonia & 1 & 1 & \\
Alveolar fistula & 0 & 1 & \\
Delirium & 0 & & \\
\hline
\end{tabular}

SVC, superior vena cava

Table 3. Recurrence patterns and causes of death

\begin{tabular}{|c|c|c|c|}
\hline & Group PN $(n=18)$ & Group PS $(n=12)$ & $p$ value \\
\hline Recurrence patterns & & & 0.791 \\
\hline Total number & $7(38.9 \%)$ & $3(25.0 \%)$ & \\
\hline Locoregional & $1(5.6 \%)$ & $0(0.0 \%)$ & \\
\hline Distant & $4(22.2 \%)$ & $2(16.7 \%)$ & \\
\hline Both & $2(11.1 \%)$ & $1(8.3 \%)$ & \\
\hline Causes of death & & & 0.270 \\
\hline Total number & $10(55.5 \%)$ & $4(33.3 \%)$ & \\
\hline Lung cancer & $4(22.2 \%)$ & $3(25.0 \%)$ & \\
\hline Other diseases & $6(33.3 \%)$ & $1(8.3 \%)$ & \\
\hline Respiratory disorders & $4(22.2 \%)$ & $0(0.0 \%)$ & \\
\hline Acute myocardial infarction & $1(5.6 \%)$ & $0(0.0 \%)$ & \\
\hline Hemorrhagic shock & $1(5.6 \%)$ & $0(0.0 \%)$ & \\
\hline Another cancer & $0(0.0 \%)$ & $1(8.3 \%)$ & \\
\hline
\end{tabular}

PS were lung cancer in four and three patients, respectively, and other diseases in six and one patient, respectively. There were no significant differences in these causes of death between the two groups $(p=0.270)$ (Table 3). In Group PN, the other diseases causing death were respiratory disorders $(n=4)$, acute myocardial infarction $(n=1)$, and hemorrhagic shock due to apart of PA ligation $(n=1)$; in Group PS, the only other cause of death besides lung cancer was another type of cancer $(n=1)$.

\section{Discussion}

Lung cancer is the leading cause of cancer-related death worldwide ${ }^{4)}$. Surgical resection is one of the mainstays for treatment of NSCLC together with chemotherapy, radiation therapy, and recent immunotherapy. Surgical treatment of NSCLC involving the proximal bronchi or PA can be challenging. Pneumonectomy is the most extensive pulmonary resection with which to ensure complete resection for these patients. However, pneumo- 


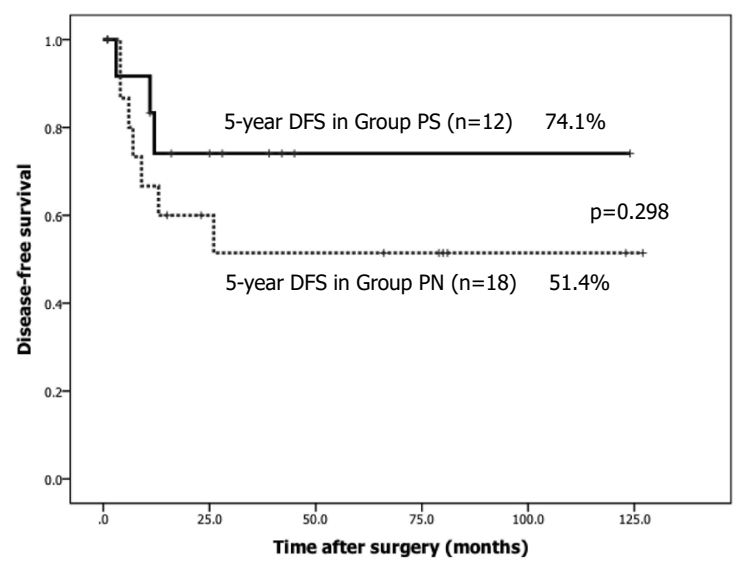

Fig. 1. Postoperative disease-free survival (DFS) between the two groups. DFS was compared between patients who underwent pulmonary function-preserving surgery (Group PS, pulmonary function-preserving surgery group, solid line) and pneumonectomy (Group PN, pneumonectomy group, dotted line). The log-rank test revealed no significant difference between them $(p=0.298)$.

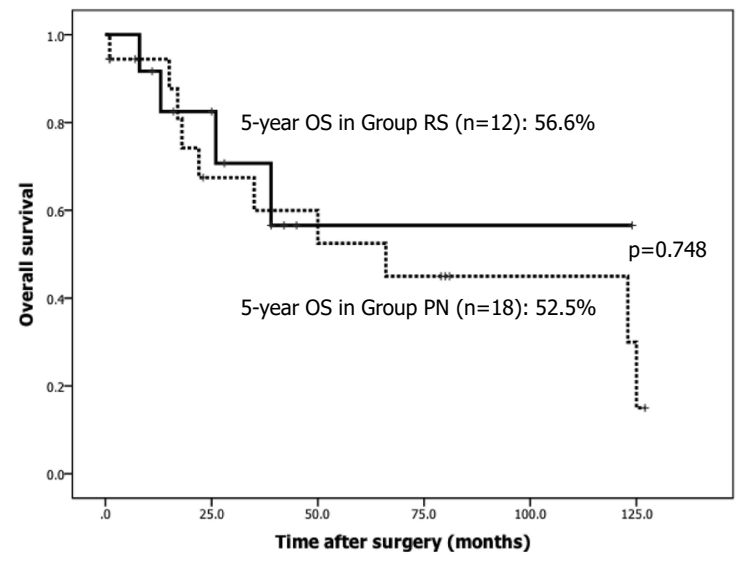

Fig. 2. Postoperative overall survival (OS) between the two groups. OS was compared between patients who underwent pulmonary function-preserving surgery (Group PS, solid line) and pneumonectomy (Group PN, dotted line). The logrank test revealed no significant difference between them $(p=0.748)$.

nectomy is associated with high complication rates, especially for patients with compromised pulmonary function. In recent years, the resectability of locally advanced lung cancer has been improving with advances in perioperative care, surgical techniques ${ }^{5-7)}$, and induction therapy ${ }^{8-10)}$, which downstages the tumors to render them resectable. Thus, avoidance of pneumonectomy can be achieved in selected patients at an early disease stage. The first sleeve lobectomy was performed by Prince-Thomas in $1942^{11)}$, and the oncologic value of lobectomy with pulmonary arterioplasty was initially reported by
Vogt-Moykopf $e t$ al. ${ }^{12)}$ in $1986 . \quad$ These procedures have since been accepted as valuable options to avoid pneumonectomy in selected patients. Many retrospective analyses have evaluated the operative mortality and morbidity of pneumonectomy and pulmonary function-preserving surgeries such as sleeve lobectomy ${ }^{1-3)}$ or PA reconstruction ${ }^{13)}$ in patients with NSCLC. In one study, the overall operative mortality associated with the surgical treatment of lung cancer in Japan was reportedly satisfactorily low, with a 30 -day mortality rate of $0.33 \%{ }^{14}$. In that survey, the 30 -day procedure-related mortality rate was $0.34 \%$ for lobectomy and $1.97 \%$ for pneumonectomy ${ }^{14}$. According to recent international reports, sleeve lobectomy or lobectomy with angioplasty can be performed with a much lower operative mortality rate $(0.0 \%-7.5 \%)$ than achieved with pneumonectomy $(4.9 \%-12.0 \%)^{13,15-17)}$.

The complication rate in our study was $33.3 \%$ in Group PS and $72.2 \%$ in Group PN and included pneumonia, hoarseness, chylothorax, pyothorax, and other conditions (Table 2). Fewer postoperative complications occurred in Group PS than PN. The incidence of postoperative complications in Group PS was comparable with that in other studies ${ }^{7,18}$. No bronchopleural fistulas occurred among 12 patients who underwent curative lobectomy with bronchoplasty or PA reconstruction, whereas the incidence was $11 \%$ in previous reports of sleeve lobectomy ${ }^{7,18)}$. On the other hand, 3 of 18 patients (16.7\%) who underwent pneumonectomy developed stump failure. Either the low blood perfusion of the blind end of the resected margin or the increased airway pressure might have influenced the higher rate of bronchopleural fistula after pneumonectomy. In Group PS, neither PA stenosis nor obstruction occurred among patients who underwent PA reconstruction. Operation-related death occurred in one patient in Group PN ; this number is comparable with that in a previous report ${ }^{19)}$. These results indicate that lobectomy with bronchoplasty or angioplasty is a safer procedure than pneumonectomy. The results of this study clearly show that pulmonary function-preserving surgery is a satisfactory treatment for lung cancer in terms of operative risk.

One possible problem associated with pulmonary function-preserving surgery for lung cancer is the potentially increased rate of local recurrence due to the remaining lung tissue. The local recurrence rate after sleeve lobectomy ranges from $8 \%$ to $23 \%{ }^{20,21)}$ and that after PA reconstruction is $26.3 \%{ }^{11)}$, whereas the local recurrence rate after pneumonectomy reportedly ranges from $10 \%$ to $14 \%^{20,21)}$. 
Pneumonectomy is therefore most likely to be a more appropriate surgical procedure for tumors involving the proximity to the hilum when performed with curative intent. In this study, however, Group PS had a somewhat higher DFS than Group PN. Local recurrence was observed in one patient (8.3\%) in Group PS and three patients (16.7\%) in Group $\mathrm{PN}$. This finding indicates satisfactory local control of central-type lung cancer by pulmonary functionpreserving surgery. There was no significant difference in DFS between the two groups. However, in Group PS, the 5-year OS rate was inferior to the 5 -year DFS rate, indicating that the prognosis in Group PS might be affected by the surgical procedure itself despite the fact that the postoperative morbidity in this group was significantly lower.

Our study showed some superior clinical aspects in Group PS compared with Group PN. However, our study had a small sample size and therefore limited power. A larger study is needed to compare the superiority and inferiority of pulmonary function-preserving surgery with those of pneumonectomy.

\section{Conclusions}

The short-term results were superior in Group PS compared with Group PN. However, the longterm results in both groups were similar. Other causes of death influenced OS in both groups, especially OS in Group PS, which might have been affected by the surgical procedure. A prospective randomized trial may help to resolve these issues.

\section{Acknowledgements}

Not applicable

\section{Conflict of Interest Disclosure}

All authors declare that they have no conflict of interest.

\section{References}

1. Bernard A, Deschamps C, Allen MS, et al. Pneumonectomy for malignant disease : factors affecting early morbidity and mortality. J Thorac Cardiovasc Surg, 121 : 1076-1086, 2001.

2. Ferguson MK, Lehman AG. Sleeve lobectomy or pneumonectomy : optional management strategy using decision analysis techniques. Ann Thorac Surg, 76 : 1782-1788, 2003.
3. Ferguson MK, Karrison T. Does pneumonectomy for lung cancer adversely influence long-term survival? J Thorac Cardiovasc Surg, 119 : 440-448, 2000.

4. Surveillance, Epidemiology, and End Results (SEER) Cancer Statistics Review, 1975-2010. http://seer.cancer.gov/csr/1975_2010. Accessed 14 June 2013.

5. Ohta M, Sawabata N, Maeda H, Matsuda H. Efficacy and safety of tracheobronchoplasty after induction therapy for locally advanced lung cancer. J Thorac Cardiovasc Surg, 125 : 96-100, 2003.

6. Lausberg HF, Graeter TP, Wendler O, Demertzis S, Ukena D, Schafers HJ. Bronchial and bronchovascular sleeve resection for treatment of central lung tumors. Ann Thorac Surg, 70 : 367-371, 2000.

7. Tedder M, Anstadt MP, Tedder SD, Lowe JE. Current morbidity, mortality, and survival after bronchoplastic procedures for malignancy. Ann Thorac Surg, 54 : 387-391, 1992.

8. Roth JA, Fossella F, Komaki R, et al. A randomized trial comparing perioperative chemotherapy and surgery with surgery alone in resectable stage IIIA non-small cell lung cancer. J Natl Cancer Inst, 86 : 673-680, 1994.

9. Roberts JR, Eustis C, Devore R, Carbone D, Choy H, Johnson D. MInduction chemotherapy increases perioperative complications in patients undergoing resection for non-small cell lung cancer. Ann Thorac Surg, 72 : 885-888, 2001.

10. Matsubara Y, Takeda S, Mashimo T. Risk stratification of lung cancer surgery : impact of induction therapy and extended surgery. Chest, 128 : 3519-3525, 2005.

11. Prince-Thomas C. Conserving resection of the bronchial tree. J R Coll Surg Edinb, 1056 ; 169186, 1942.

12. Vogt-Moykopf I, Fritz TH, Meyer G, Bulzerbruck H, Daskos G. Bronchoplastic and angioplastic operation in bronchial carcinoma : long-term results of a retrospective analysis from 1973 to 1983 . Int Surg, 71 : 211-220, 1986.

13. Ma Q, Liu D, Guo Y, et al. Surgical techniques and results of the pulmonary artery reconstruction for patients with central non-small cell lung cancer. Cardiothorac Surg, 8 : 219, 2013.

14. Committee for Scientific Affairs, The Japanese Association for Thoracic Surgery, Nasuda M, Kuwano H, Okumura M, Arai H, Endo S, Doki Y, et al. Thoracic and cardiovascular surgery in Japan during 2013 : annual report by The Japanese Association for Thoracic Surgery. Gen Thorac Cardiovasc Surg, 63 : 670-701, 2015.

15. Stoelben E, Suerbrei W, Ludwig C, Hasse J. Tumor stage and early mortality for surgical resections in lung cancer. Arch Surg, 388 : 116-121, 
2003.

16. Deslauriers J, Gregoire J, Jacques LF, Piraux M, Guojin L, Lacasse Y. Sleeve lobectomy versus pneumonectomy for lung cancer : a comparative analysis of survival and sites or recurrences. Ann Thorac Surg, 77 : 1152-1156, 2004.

17. Ludwig C, Stoelben E, Olschewski M, Hasse J. Comparison of morbidity, 30-day mortality, and long-term survival after pneumonectomy and sleeve lobectomy for non-small cell lung carcinoma. Ann Thorac Surg, 79 : 968-973, 2003.

18. Weisel RD, Cooper JD, Delarue NC, Theman TE, Todd TR, Pearson FG. Sleeve lobectomy for carcinoma of the lung. J Thorac Cardiovasc Surg,
$78: 839-844,1979$.

19. Ginsberg RJ, Hill LD, Eagan RT, et al. Modern thirty-day operative mortality for surgical resections in lung cancer. J Thorac Cardiovasc Surg, 86 : 654-658, 1983.

20. Okada M, Yamagishi H, Satake S, et al. Survival related to lymph node involvement in lung cancer after sleeve lobectomy compared with pneumonectomy. J Thorac Cardiovasc Surg, 119 : 814-819, 2000.

21. Mehran RJ, Deslauriers J, Piraux M, Beaulieu M, Guimont C, Brisson J. Survival related to nodal status after sleeve resection for lung cancer. J Thorac Cardiovasc Surg, 107 : 888-893, 1994. 